\title{
ULTRAVIOLET LIGHT-INDUCED RECOMBINATION
}

\author{
Robert B. Helling \\ Department of Botany \\ University of Michigan \\ Ann Arbor, Mich. 48104
}

Received October 8,1973

Stimulation of transduction in Escherichia coll by ultraviolet irradiation of the transducing phage $P 1$ requires the uvrA-uvrB nuclease but not the uvrC product or DNA polymerase $I$. It is hypothesized that the first step in "normal" recombination can be bypassed by any procedure generating singlestranded ends of DNA (as, for example, by uvrA-uvrB nuclease activity).

Stimulation of recombination by ultraviolet (UV) irradiation was discovered by Jacob and Wollman ${ }^{1}$ using the phage $\boldsymbol{\lambda}$. Subsequently recombination in both prokaryotic and eukaryotic organisms has been shown to be stimulated by UV light and other agents acting on DNA.

A close relationship between the repair of damaged DNA and recombination was established directly by the isolation of rec mutants of Escherichia coll which are defective in both functions ${ }^{2}$. Other types of mutants deficient in DNA-repair have been isolated, but most of these exhibit normal recombination frequencies. The role of each of these "repair" genes in UV-stimulated recombination has not been determined.

In my work the effect of UV light on recombination in mutants of $\underline{E}$. coli deficient in excision-repair of DNA has been examined. uvrA and uvrB mutants lack an endonuclease which makes a single-strand break in DNA containing a UVinduced pyrimidine dimer ${ }^{3}$. uvrC mutants have this activity ${ }^{3}$ but are unable to make the second break which releases an oligonucleotide containing the dimer ${ }^{4}$. DNA polymerase I is believed to replace the DNA lost by excision; this enzyme is lacking in polA mutants ${ }^{5}$. DNA ligase is believed to complete excisionrepair by making the last phosphodiester linkage.

The conversion of abortive (potential) to complete transductants provides a convenient and sensitive assay for UV-stimulated recombination ${ }^{6}$. Usually less than $10 \%$ of the cells which receive a given gene from a transducing phage form stable transductants containing that gene. Following UV-irradiation, the number of complete transductants increases. This is a consequence of increased recombination which converts abortive to stable transductants ${ }^{7}$. Certain mutants of $\underline{E} \cdot$ coli $^{6}$, Salmonella typhimurium ${ }^{8}$, and Streptococcus pyogenes ${ }^{9}$ defective in DNA-repair fail to exhibit this UV-stimulated conversion.

The results of the experiments presented here show that in transduction, 


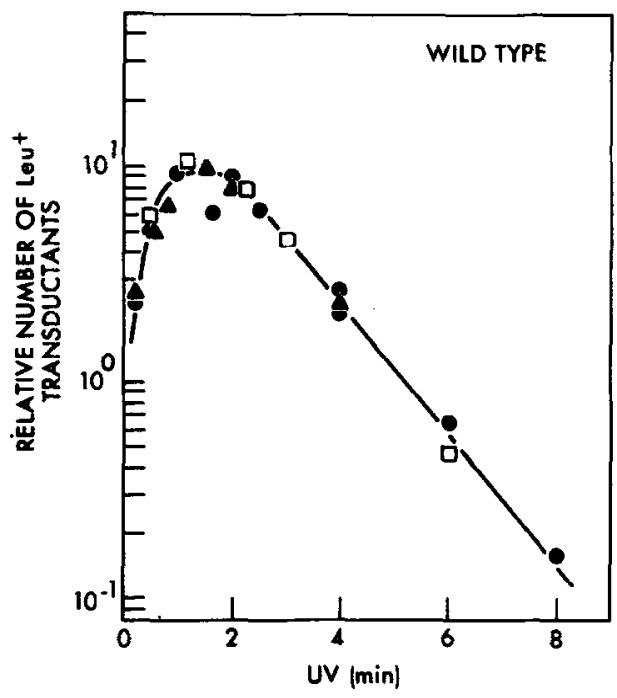

Figure 1. Stimulation of transduction of $\mathrm{KH}^{21}$ to Leu ${ }^{+}$by UV-irradiation of the transducing phage.' Cells were harvested from exponential growth "In minimal g1ycerol medium and infected at a multiplicity of 0.2 in medium $X$ supplemented with $0.005 \%$ thymine and $0.0001 \%$ biotin. (Medium $X$ consists of $0.012 \mathrm{M} \mathrm{MgSO}_{4}$, $0.025 \mathrm{M} \mathrm{CaCl}_{2}, 2 \%$ ammonium acetate, $4 \%$ tryptone broth and $0.4 \%$ glycerol.) After $40 \mathrm{~min}$ in an ice bath, followed by $40 \mathrm{~min}$ at room temperature, aliquots were plated in melted agar medium selective for Leu transductants. After hardening the plates were incubated 3 days at $37 \mathrm{C}$ before counting. Results from 3 experiments are plotted.

WV-induced recombination is initiated by the first step in the excision-repair pathway. The product of this step, DNA with a single-strand nick near a dimer, can either enter the reaction sequence terminating in recombination, or instead, continue in the excision-repair pathway.

\section{MATERIALS AND METHODS}

KH21 is leuB1 lacY bio thyA strA metE rha ma1B (a K12 strain). Derivatives containing the mutations uvrA6 $^{10}$, polA1 ${ }^{5}$, or the uvrB mutation from E. coli Bs1 ${ }^{6}$ were obtained by cotransduction with ma1B, metE or bio. The mutant transductants were identified by UV-sensitivity and verifled by transducing the mutation back to $\mathrm{KH} 21$. AB2497 is thi-1 h1s-4 proA2 1eu-6 thr-1 thyA17 thyR11 $\underline{m t 1-1}$ xy1-5 ara-14 galk2 lacY1 str-31 tsx-33 sup-37 (amber). Ab2498 is its uvrC34 derivative ${ }^{10}$. These two strains came from the collection of K.C. Smith via D. Youngs.

Media and general methods including UV-irradiation (incident dose-rate 1330 ergs $/ \mathrm{mm}^{2} / \mathrm{min}$ ) and transduction with phage Plbt have been described ${ }^{6}$.

\section{RESULTS}

Stimulation of recombination by irradiation of the transducing phage is shown in Fig. 1. Assuming 0.13 pyrimidine dimers per phage genome $\left(10^{5}\right.$ nucleo- 


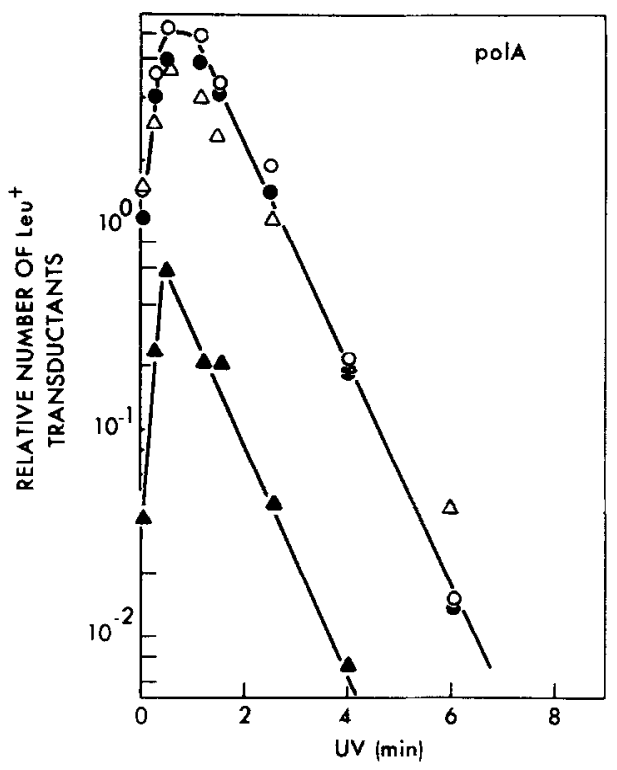

Figure 2. Influence of recipient's growth condition on frequency of normal and UV-induced recombination. The recipient was polA metE $\mathrm{KH}^{+}$. The influence of growth medium on KH2l was similar. The recipient was harvested from exponential growth $\left(3 \times 10^{8} \mathrm{cells} / \mathrm{ml}\right)$ in minimal-glycerol medium $(0)$ or L-broth $(\Delta)$, or from stationary phase (about $1.5 \times 10^{9}$ cells/m1) in minimal-glycerol (o), or L-broth $(\Delta)$.

tide pairs) per erg per $\mathrm{mm}^{2} 4,6,11,12$ the maximum number of transductants is obtained at a UV dose equivalent to about 250 dimers per phage particle ( 1 1/2 min) or about 1 dimer per 400 nucleotide pairs.

Influence of the growth medium. The medium in which the reciplent was grown prior to infection had no effect on the shapes of the dose-response curves. However, the number of transductants recovered was much lower when cells exponentially growing in a rich medium were used rather than cells from minimal medium or cells grown into stationary phase in rich medium (Fig. 2). In subst?uent experiments cells were harvested from exponential growth in mintmal medium.

UV-stimulated recombination requires $\underline{\mathrm{uvrA}}^{+}$and $\underline{\mathrm{uvrB}}{ }^{+}$but not $\underline{\mathrm{urC}}^{+}$or polA. Cells deficient in the dimer-specific uvrA-uvrB endonuclease are a1so deficient in UV-stimulated recombination (Fig. 3). The small stimulus observed shows that some other enzyme can partia11y replace the uvrA-uvrB nuclease, but most UV-induced recombination is dependent on this function. The response of the double mutant uvrA-uvrB was similar to that of the single mutants (not shown), so the small stimulus is unlikely to be due to leakiness of the individual uvrA and uvrB mutations. 


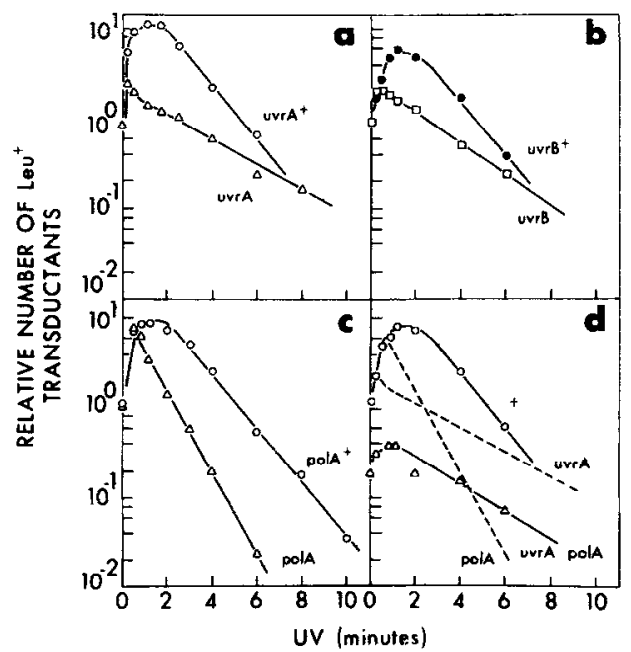

Figure 3. The roles of uvrat, uvrB, and polA ${ }^{+}$genes in induced transduction. Mutant recipients were a) uvrA mal $^{+} \mathrm{KH}_{21}$; b) uvrB bio $^{+} \mathrm{KH} 21$; c) polA met $\mathrm{K}^{+} \mathrm{KH} 21$; d) uvrA po1A met ${ }^{+}$mal $^{+} \mathrm{KH} 21$. Numbers plotted are relative to those of the wildtype recipient.

Contrary to the behavior of the mutants lacklng the first step in excisionrepair, recombination in polA mutants is greatly stimulated by UV-irradiation to the transducing phage (Fig. 3). In the uvrC mutant and its uvrC ${ }^{+}$parent, the frequency of transduction using untreated phage is higher than in $\mathrm{KH} 21$ and derivatives of $\mathrm{KH} 21$ (Fig.4). The basis for the difference has not been determined. However, UV-treatment of the phage increases the frequency 3- to 5fold in both the mutant and the uvrC ${ }^{+}$parent. In the mutant, maximal stimulation is achieved by a UV dose lower than required with wild type cells.

These results show that UV-induced recombination requires a functiona1 uvrA-uvrB nuclease, but neither the uvrC $^{+}$function nor DNA polymerase I. Double mutants uvrA po1A and uvrB polA were constructed and tested in recombination to check this conclusion. As expected the uvrA polA recipient behaves like a uvrA mutant in that little recombination is induced by UV light (Fig. 3). (Fewer recombinants were obtained even without UV treatment, probably reflecting the high proportion of nonviable cells usually seen in uvrA polA strains ${ }^{13}$.) Unexpectedly, the uvrB polA strain did not grow on complex media, although it could grow on minimal media. Shizuya and Dykhuizen ${ }^{14}$ found a similar result, using uvrB point mutations; uvrB polA double mutants constructed from uvrB deletions, and a temperature-sensitive polA mutation are invariably lethal at the nonpermissive temperature. In my experiments, when uvxB polA mutants were used as reciplents, no transductants were recovered. In view of the viability effects noted above it is plausible that this is a secondary effect, 


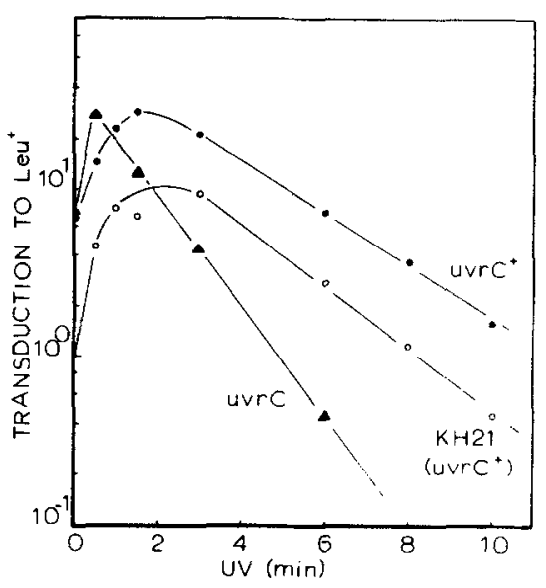

Figure 4. The role of the uvrC $^{+}$gene in induced transduction. Strains were $\mathrm{AB} 2497, \mathrm{AB} 2498$, and $\mathrm{KH} 21$. An uncalibrated UV-source was used for this experiment.

resulting from lethality in cells undergoing recombination rather than inadequacy in the recombination process itself.

The loss of transductants. Comparison of the final inactivation rates shows that inactivation by UV-irradiation of the phage is greatest in polA and uvrC strains and least in uvrA and uvrB strains. I provisionally explain the differences in final slopes as follows. In the wild-type cell, the product of the uvrA-uvrB enzyme can a) enter a pathway leading to recombination; or b) continue in the excision-repair pathway, thus restoring the normal DNA structure but failing to form a UV-Induced recombinant; or c) undergo degradation by other nucleases, thus failing to produce any recombinants whatsoever.

Although uvrA or uvrB mutants show little stimulation of recombination by UV light, the "normal" formation of recombinants is not inhibited by excisionrepair, nor are normal recombinants lost by degradation during such repair. Thus the number of transductants in uvrA and uvrB cells shows a slower decline with increasing UV-dose because a pathway leading to degradation as well as stimulation is blocked.

The uvrC and polA strains show an increased rate of inactivation of transduction relative both to the uvrA or uvrB strains and to the wild-type. Thus as the distance between adjacent dimers decreases with increasing dose rate, more of the potential recombinant strands produced by the uvrA-uvrB endonuclease are destroyed without completing efther UV-induced recombination or normal recombination than in the wild-type.

\section{DISCUSSION}

The system for studying recombination described here has several advan- 
tages. The DNA in the phage can be irradiated and subsequently used to infect an undamaged host cel1. Recombination is limited to a defined segment of bacterial DNA carried by the transducing phage and the homologous region of the chromosome. The recombination depends entirely on host enzymes because a transducing particle contains no phage genes 15 .

What is the nature of the product of the uvrA-uvrB endonuclease which makes the DNA active in recombination? Is the single-strand nick sufficient? No, because single nicks mede by ${ }^{32} \mathrm{P}$-decay ${ }^{16,17}$, or X-irradiation ${ }^{18}$ fall to stimulate transduction. Instead recombination may result from the appearance of a singlestranded end. The end with the dimer may not pair properly, and the region of single-stxandedness may be extended by transcription. This possibility is intuitively appealing for it provides a logical mechanism for the pairing of homologous strands. The free single-strand can anneal with a complementary strand in the chromosome which is unpatred as the result of replication, transcription, repair, or unwinding by an enzyme of normal recombination.

This paper has shown that the exclsion-repair genes $\underline{u v r A}^{+}$and uvr $^{+}$are required for most UV-stimulated transduction. Experiments involving repair genes not in the excision-repair pathway show that the recA product (but not the recB-recC nuclease) is also needed. The roles of these and other genes in UVstimulated recombination will be discussed in a subsequent paper. A11 of the results are consistent with the concept that the initial step (s) in normal recombination can be bypassed by any procedure generating single-stranded ends, but the overall process is still dependent on subsequent steps involving recA ${ }^{+}$.

I thank J. Cairns and D. Youngs for strains, and H. Boyer for his hospitality while this was written. This work was supported by a grant from the Michigan Memorial-Phoenix Project.

\section{REFERENCES}

1. Jacob, F., and E.L. Wollman. 1955. Ann. Inst. Pasteur, 88:724

2. Clark, A.J., and A.D. Margulies. [965. Proc. Nat1. Acad. Sc1. USA, 53:451.

3. Grossman, L. 1973. In DNA Synthesis in Vitro (ed. R.D. We1ls and R.B. Inman), Univ. Park Press, Baltimore; and personal communication, Feb. 1973.

4. Kato, T. 1972. J. Bacterio1., 112:1237.

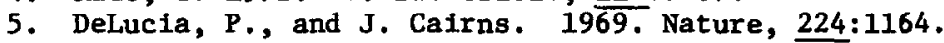

6. Helling, R.B. 1969. J. Bacteriol., 100:224.

7. Benzinger, R., and P.E. Hartman. 1962. Virology, 18:614.

8. Takebe, H. 1968. Biochem. Biophys. Res. Commun., 31:938.

9. Malke, H. 1969. Personal communication.

10. Howard-Flanders, P., R.P. Boyce, and L. Therlot. 1966. Genetics, 53:1119.

11. Smith, K.C. 1969. Mutat. Res., 8:481.

12. Shlaes, D.M., J.A. Anderson, and S.D. Barbour, 1972. J. Bacter1o1., 111:723.

13. Capaldo-Kimbal1, F., and S.D. Barbour. 1971. J. Bacter1o1., 106:204.

14. Shizuya, H., and D. Dykhuizen. 1972. J. Bacter101., 112:676.

15. Ikeda, A., and J. Tomizawa. 1965. J. Mo1. Blol., 14:85.

16. Hartman, P.E., and W.A. Kozinsk1. 1962. Virology, 17:233.

17. Tomizawa, J., and H. Ogawa. 1968. Cold Spring Harbor Symp. Quant. Biol., $33: 243$.

18. Takebe, H.K., and P.E. Hartman. 1962. Vlology 17:295. 